\title{
Evaluation of mass transfer coefficients in biotrickling filters: experimental determination and comparison to correlations \\ Antonio D. Dorado ${ }^{1}$, Ginesta Rodríguez ${ }^{1}$, Gemma Ribera ${ }^{1}$, Anna Bonsfills ${ }^{1}$, David Gabriel $^{2}$, Javier Lafuente ${ }^{2}$ and Xavier Gamisans ${ }^{1}$
}

\begin{abstract}
Overall mass transfer coefficients $\left(\mathrm{K}_{\mathrm{G}} \mathrm{a}\right.$ and $\left.\mathrm{K}_{\mathrm{L}} \mathrm{a}\right)$ were determined experimentally for four different-nature packing materials used in gas-phase biotrickling filters. A simple methodology based on overall mass balances and following a standard procedure allowed to calculate the mass transfer coefficients at different operating conditions corresponding to usual biotrickling filtration situations. Results showed an increase of mass transfer resistance when increasing the empty bed residence time (EBRT) of the reactor for all packing materials. Experimental results were fitted to existing and wellaccepted correlations used in conventional biofilters or biotrickling filters modelling. Comparison between experimental and theoretical data showed huge discrepancies. Simple correlations for the experimental data obtained in this study were also suggested.
\end{abstract}

\footnotetext{
${ }^{1}$ Department of Mining Engineering and Natural Resources, Escola Politécnica Superior d'Enginyeria de Manresa, Universitat Politécnica de Catalunya.

2 Department of Chemical Engineering, Escola Técnica Superior d'Enginyeria, Universitat Autónoma de Barcelona.
}

Correspondence: Dr. Ing. X. Gamisans (xavierg@emrn.upc.edu), EPSEM, Universitat Politécnica de Catalunya (UPC). Bases de Manresa, 61-73, 08242 Manresa, Spain. 


\section{INTRODUCTION}

Biofiltration is a relatively new technology for gaseous streams treatment that has gained popularity in the last decades. In general, the suitability of either biofilters or biotrickling filters for the abatement of a wide range of pollutants and conditions is well known [1]. The main capabilities of these systems are closely related to aspects regarding to both interfacial mass transfer and biodegradation kinetics. Traditionally the latter issue has received much attention and the number of experimental studies at different scale is considerably huge. Conversely, mass transfer resistance has received little attention from the scientific community dealing with biofilter systems, although this is a quite probable phenomenon to occur.

Only a few works have considered mass transfer resistance in the gas phase to describe pollutant removal for both biofilters and biotrickling filters [2]. In general, modeling studies have used a mass transfer coefficient for the transport from the gas phase to the biofilm [3-7].

Mass transfer coefficients, used for characterizing phase resistances, are usually calculated by means of empirical correlations. Onda's correlation [8] has been commonly used for calculating mass transfer coefficients for gas and liquid phases. In this equation, coefficient values are calculated as a function of pollutant physicochemical properties, reactor and packing material characteristics and operational parameters. However, this and other existing empirical correlations were created for specific (and sometimes older) packing materials that today are no longer used. For this reason, it is possible that traditional correlations are not suitable for modern packing materials used in biofiltration. It has been noticed by a few authors that Onda's equations predict considerably high values for mass transfer coefficients. This has led to some authors to incorporate correction factors into empirical correlations [9]. It is noteworthy to point out that all these correlations were mainly developed for wet scrubbers operating at conditions quite different from those found in biofiltration.

In addition, it is not clear where is located the predominant mass transfer resistance due to the low gas and liquid velocities commonly used in biofilters and biotrickling filters. As pointed out by Kim and Deshusses [10], fluids velocity in biofilters or biotrickling 
filters might be considerably different from those found in wet scrubbers. This promotes at least some doubts regarding the suitability of chemical scrubbers derived correlations for mass transfer coefficients predictions in biofilters or biotrickling filters.

On the other hand, experimental lab-scale results strongly depend on the experimental setup characteristics and/or limitations (fluids distribution, evaporation effects, column and packing particles diameters ratio). In addition, the analytical methods should be simple to ensure that no bias arises from the use of complex experimental systems. In this sense, some works have been developed to establish a standard procedure for mass transfer studies in gas-liquid mass transfer systems [11].

Wang et al. [12] performed a comprehensive summary of the models found in the literature for both mass transfer coefficients and effective interfacial areas. The authors concluded that more research works have to be done in the near future since there is still a lack of understanding of the complex phenomena occurring in mass transfer in gasliquid contactors.

At present, Kim and Deshusses [13] have performed the only one comprehensive study devoted to mass transfer in biofilters and biotrickling filters. They designed a set of experiments to experimentally evaluate both individual gas and liquid mass transfer coefficients. Their study covered a variety of materials, mainly inorganic, and a wide range of operating conditions. Afterwards they proposed a set of parameters corresponding to an empirical correlation based on Onda's equations for those materials.

The aim of this study is to demonstrate the need of an accurate evaluation of mass transfer parameters for biofilters instead of using empirical correlations. For this purpose, a simple methodology is presented for the experimental determination of overall mass transfer coefficients. The method involves the possibility of using the same pollutant to be biodegraded instead of the common acid-base reaction system (e.g. carbon dioxide-sodium hydroxide) for the experimental tests. In this sense, toluene (a usual model compound in biofiltration) was used for the absorption tests.

\section{MATERIALS AND METHODS}

\subsection{Experimental Setup}


After a literature review of different methods for the experimental determination of mass transfer coefficients, the procedure described by Heymes et al. [14] was adopted due to its simplicity. The experimental determination of mass transfer coefficients has been performed in a lab-scale pilot plant (Figure 1) consisting of a PVC column $(4.7 \mathrm{~cm}$ inner diameter, $67 \mathrm{~cm}$ height). Gas flow was supplied by means of a mass flow controller (Bronkhorst High-Tech, Netherlands) prior to its pre-humidification. Gas flowrate was adjusted to provide empty bed residence time values (EBRT) from 6 to 100 s. A secondary air stream was pumped by a peristaltic pump (Mod. L/S, Masterflex) into a glass bubbler containing pure liquid toluene (Panreac 99.5\%). Both gaseous flowrates were mixed and the resulting gas mixture was fed from the bottom of the reactor in up-flow mode.

Toluene concentration in air was measured at the inlet and outlet of the reactor by an on-line photo ionization detector (Photovac 2020, $\pm 2 \mathrm{ppm}$ accuracy). The detector was connected to a computer through the RS232 port for continuous data collection. Tap water was sprinkled by a high performance hollow cone nozzle (Bete WL1) located at the top of the column. The liquid was stored in a small tank ( $1 \mathrm{~L}$ volume) and recirculated by a membrane pump (Magdos LT-10). Toluene concentration in the aqueous phase was monitored by sampling the inlet and the outlet of the reactor at regular intervals. Toluene content in water was determined by UV-VIS spectrophotometer (Perkin-Elmer, $\pm 0.1 \%$ accuracy) at 255 to $265 \mathrm{~nm}$ wavelength.

\subsection{Packing materials}

Four different packing materials have been used in this study for testing the methodology described below. Materials selected were clay pellets (STA, Spain), lava rock (Ampans Garden, Spain), polyurethane foam (PUF) (Recticel Iberica S.L., Spain) and stainless steel Dixon rings (Afora S.A., Spain). The first and the second materials are commonly used in biotrickling filters or even in conventional biofilters [1]. The third and fourth materials are in general used mainly in packed towers for absorption systems, despite polyurethane foam has been used also in biofiltration [15].

Characterization of packing materials was carried out according to standard methods [16]. The following properties were compared in each case: packed bed porosity, and 
specific surface area, density and water holding capacity of the packing material. Packing material density was determined by the BET technique in a Micromeritics (model Tristar 3000) apparatus. Specific surface areas were obtained either from the material suppliers (Polyurethane foam and Dixon rings) or by direct measurement based on physical dimensions. For the latter, the following equation was used:

$\mathrm{a}=\frac{6(1-\varepsilon)}{\varphi \mathrm{D}_{\mathrm{p}}}$

Where $\varphi$ is the sphericity factor (0.95 and 0.89 for clay pellets and lava rock respectively).

\subsection{Experimental procedure}

In order to proceed minimizing the most common errors found in mass transfer studies, Hoffmann et al. [11] suggestions for the standardization of mass transfer measurements were followed. In this sense, experimental tests (24 suitable for numerical treatment from a total of 50) were performed at room temperature $\left(23 \pm 2{ }^{\circ} \mathrm{C}\right)$ under quasiisothermal conditions. Selected packing materials for the study were screened ensuring a column diameter to particle diameter ratio above 10 (minimization of wall effects). Prior to the absorption column entrance, inlet air was passed through a humidification column to avoid evaporation and, thus, avoiding interfacial solvent mass transfer. A gas distribution plate $\left(\approx 150\right.$ drip points $\left./ \mathrm{m}^{2}\right)$ and a high performance hollow cone liquid nozzle were used for uniform gas and liquid distribution. Moderate to high gas-phase toluene concentrations (1000-2000 $\mathrm{ppm}_{\mathrm{v}}$ ) were applied to ensure mass transfer along the whole column height. Absolute pressure during the experiments was kept at 1.2 bar.

The system acted as a differential absorption column in which the water phase was continuously recirculated from the bottom to the top of the column. The system was initially loaded with a known amount of pure water and the membrane pump was connected in order to ensure the complete wetting of the packing material. Liquid phase velocity was kept at approximately $2 \mathrm{~m} / \mathrm{h}$, a typical value in biotrickling filters [1]. After a constant liquid hold-up is observed (by means of an optical level sensor in the liquid storage tank), toluene-air mixtures were passed through the column upwards. 
Different gas velocities $(0.00025$ to $0.05 \mathrm{~m} / \mathrm{s})$ were applied corresponding to typical EBRT for both conventional biofilters and biotrickling filters (6 to $100 \mathrm{~s}$ ). Toluene gas concentration was continuously monitored on-line for both the inlet and the outlet of the column, while toluene aqueous concentration in the liquid phase was measured off-line every 5 minutes as described above. Each test finished when both the liquid and gas toluene concentration remained constant (considering $\pm 5 \%$ of the final value oscillations as acceptable for steady-state conditions)

Experimental data was used to verify the mass balance for the whole column. Figure 2 shows the nomenclature and the double-film concept used in this study. Since there is no clear evidence that operating conditions in biotrickling filters allow omitting any phase resistance this is the system model considered. Thus, a mass balance for the system described is derived straightforward using the concept of global mass transfer coefficient $\left(\mathrm{K}_{\mathrm{G}}\right.$ and $\left.\mathrm{K}_{\mathrm{L}}\right)[14]$.

$$
\begin{gathered}
Q_{G}\left(C_{G, \text { in }}-C_{G, \text { out }}(t)\right)=K_{L} a V_{C} \frac{\left(\frac{C_{G, \text { in }}}{H}-C_{L, \text { out }}(t)\right)-\left(\frac{C_{G, \text { out }}(t)}{H}-C_{L, \text { in }}(t)\right)}{\ln \left(\frac{\frac{C_{G, \text { in }}}{H}-C_{L, \text { out }}(t)}{\frac{C_{G, \text { out }}(t)}{H}-C_{L, \text { in }}(t)}\right)} \\
\mathrm{Q}_{\mathrm{G}}\left(\mathrm{C}_{\mathrm{G}, \text { in }}-\mathrm{C}_{\mathrm{G}, \text { out }}(\mathrm{t})\right)=\mathrm{K}_{\mathrm{G}} \mathrm{aV}_{\mathrm{C}} \frac{\left(\mathrm{C}_{\mathrm{G}, \text { in }}-\mathrm{HC}_{\mathrm{L}, \text { out }}(\mathrm{t})\right)-\left(\mathrm{C}_{\mathrm{G}, \text { out }}(\mathrm{t})-\mathrm{HC}_{\mathrm{L}, \text { in }}(\mathrm{t})\right)}{\ln \left(\frac{\mathrm{C}_{\mathrm{G}, \text { in }}-\mathrm{HC}_{\mathrm{L}, \text { out }}(\mathrm{t})}{\mathrm{C}_{\mathrm{G}, \text { out }}(\mathrm{t})-\mathrm{HC}_{\mathrm{L}, \text { in }}(\mathrm{t})}\right)}
\end{gathered}
$$

The Henry's law constant $(\mathrm{H})$ was considered constant along the experiments since temperature was kept almost constant as stated above. The dimensionless $\mathrm{H}$ value used was adapted from Dorado et al. [4]. It is clear that this balance, which uses the mean logarithmic concentration concept, allows evaluating both global mass transfer coefficients $\mathrm{K}_{\mathrm{G}}$ a or $\mathrm{K}_{\mathrm{L}}$ a from the unsteady state data collected in each experiment. In this sense, the linearization of equations (2) and (3) is straightforward as follows:

$\mathrm{Q}_{\mathrm{G}}\left(\mathrm{C}_{\mathrm{G}, \text { in }}-\mathrm{C}_{\mathrm{G}, \text { out }}(\mathrm{t})\right)=\mathrm{K}_{\mathrm{L}} \mathrm{af}_{1}(\mathrm{C})$ 
and

$$
\mathrm{Q}_{\mathrm{G}}\left(\mathrm{C}_{\mathrm{G}, \text { in }}-\mathrm{C}_{\mathrm{G}, \text { out }}(\mathrm{t})\right)=\mathrm{K}_{\mathrm{G}} \mathrm{af}_{2}(\mathrm{C})
$$

where:

$$
\mathrm{f}_{1}(\mathrm{C})=\mathrm{V}_{\mathrm{C}} \frac{\left(\frac{\mathrm{C}_{\mathrm{G}, \text { in }}}{\mathrm{H}}-\mathrm{C}_{\mathrm{L}, \text { out }}(\mathrm{t})\right)-\left(\frac{\mathrm{C}_{\mathrm{G}, \text { out }}(\mathrm{t})}{\mathrm{H}}-\mathrm{C}_{\mathrm{L}, \text { in }}(\mathrm{t})\right)}{\ln \left(\frac{\frac{\mathrm{C}_{\mathrm{G}, \text { in }}}{\mathrm{H}}-\mathrm{C}_{\mathrm{L}, \text { out }}(\mathrm{t})}{\frac{\mathrm{C}_{\mathrm{G}, \text { out }}(\mathrm{t})}{\mathrm{H}}-\mathrm{C}_{\mathrm{L}, \text { in }}(\mathrm{t})}\right)}
$$

and

$$
\mathrm{f}_{2}(\mathrm{C})=\mathrm{V}_{\mathrm{C}} \frac{\left(\mathrm{C}_{\mathrm{G}, \text { in }}-\mathrm{HC}_{\mathrm{L}, \text { out }}(\mathrm{t})\right)-\left(\mathrm{C}_{\mathrm{G}, \text { out }}(\mathrm{t})-\mathrm{HC}_{\mathrm{L}, \text { in }}(\mathrm{t})\right)}{\ln \left(\frac{\mathrm{C}_{\mathrm{G}, \text { in }}-\mathrm{HC}_{\mathrm{L}, \text { out }}(\mathrm{t})}{\mathrm{C}_{\mathrm{G}, \text { out }}(\mathrm{t})-\mathrm{HC}_{\mathrm{L}, \text { in }}(\mathrm{t})}\right)}
$$

This procedure was used for checking experimental mass balances.

\section{RESULTS AND DISCUSSION}

\subsection{Characterization of packing materials}

Main characteristics of packing materials tested are shown in Table 1. Materials selected covered a wide range specific surface areas and packed bed porosities. Both are key parameters related to mass transfer

Clay pellets provided the lowest bed porosity after being screened to obtain a homogeneous particle size of $4-5 \mathrm{~mm}$. In this sense, the relatively high specific surface area found for clay pellets might be inefficient in terms of mass transfer because of the low bed porosity of this material. It is worth mentioning that a very common material used in biofiltration as lava rock showed the lowest specific surface area with moderate bed porosities. As expected, polyurethane foam provided the highest bed porosity. Polyurethane foam is a light, open-pore foam with a large void volume of around 90-93\% [17] and relatively high surface area. Dixon rings provided the highest specific surface area and relatively high bed porosities. Additionally, since toluene is a moderately hydrophobic compound, the water holding capacity should play also an important role in the absorption capacity of each material. Although there is not a material that can be regarded as the best (a priori) for mass transfer purposes, Dixon rings and polyurethane 
foam seem to be more adequate than clay pellets and lava rock in terms of the evaluated properties.

\subsection{Experimental results}

Figure 3 shows an example of raw data obtained during one of the several experimental tests performed. In this case, data correspond to polyurethane foam as packing material operating at an EBRT of 35 seconds. As expected in countercurrent differential operation, both liquid and gas outlet concentrations rise continuously from an initial close-to-zero value. It is noteworthy that from the very beginning the gas outlet concentration was above zero, meaning that the system is not so much effective as scrubber. In the same way, the initial outlet liquid concentration was also relatively high. This was caused by the sampling time needed for obtaining a representative sample of the column. As can be also observed in Figure 3, after 70 minutes constant concentrations were observed for both phases, meaning that a steady state is reached and, thus, the experiment is finished. Therefore, experiments length was 70 minutes in the majority of cases.

The numerical procedure previously described was used for global mass transfer coefficients determination. Figure 4 shows a typical figure obtained and the corresponding coefficient values derived. Good determination coefficients $\left(r^{2}>0.95\right.$ in all cases) were obtained for all the experimental tests performed. These values confirm that the mass balance equation used represents satisfactorily the column behavior.

Experimental $\mathrm{K}_{\mathrm{G}} \mathrm{a}$ values obtained for the four packing materials described above are shown in figure 5 . Since $\mathrm{K}_{\mathrm{L}}$ a values can be obtained from $\mathrm{K}_{\mathrm{G}} \mathrm{a}$ ones, the discussion will be performed only considering $\mathrm{K}_{\mathrm{G}} \mathrm{a}$ data. It is clear that only slight differences were observed at EBRT values higher than 40 seconds. Conversely, the most important differences between materials behavior were found at EBRT below 20 seconds. This is in agreement with the fluid dynamics within the column. An increase on the gas flow rate (i.e. decreasing the EBRT), implies also an increase on the turbulence within the packed bed. This turbulence improved the interfacial mass transfer and, thus, the global mass transfer coefficient increased drastically. Dixon rings showed the highest mass transfer coefficients values (i.e. the lower mass transfer resistance), followed by PUF 
and lava rock. Values for clay pellets were the lowest, meaning that mass transfer limitations are more possible to occur when using this material at low EBRT. Packed bed porosity might be a possible explanation of this. However, one would expect that increasing the porosity would decrease the interstitial velocity and, thus, decrease the mass transfer coefficient. This was not observed clearly during this study.

The practically inexistent differences between materials at high EBRT values are related to the fact that in these conditions the toluene diffusion in the gas phase is probably the predominant process in the global interfacial mass transfer process. This means that neither the liquid side resistance nor the packing materials characteristics affect the global system performance. Therefore, neglecting gas phase resistance at high EBRTs in modeling studies might be an important error source.

A simple observation of the experimental results allows thinking of plain mathematical expressions for data fitting. In this sense, an inverse proportionality relationship between the EBRT and the mass transfer coefficient could be presumably the most suitable. Experimental data can be easily fitted to simple empirical equations such as the following:

$$
\mathrm{K}_{\mathrm{G}} \mathrm{a}=\mathrm{b}_{1} \mathrm{EBRT}^{\mathrm{b}_{2}}
$$

Table 2 shows the values for coefficients $b_{1}$ and $b_{2}$ obtained by non-linear regression for the four packing materials tested. $K_{\mathrm{G}}$ a values calculated with $b_{1}$ and $b_{2}$ coefficients showed a proper description of experimental data (Figure 5). Thus, from the determination coefficients obtained $\left(\mathrm{r}^{2}\right)$ it is possible to ensure that such simple equations are perfectly suitable for the description of the relationship between fluid dynamics and mass transfer. It is noteworthy to point out the almost close to -1 value of exponent $b_{2}$ for most of the packing materials.

\subsection{Data fitting to existing correlations}

The majority of literature papers related to mass transfer in biofilters assume that transport resistance is mainly caused by one of either gas or liquid phase. However, it is hard to find a set of conditions in which this is completely true. For instance, Rejl et al. [18] pointed out that only at gas velocities $\geq 0.5 \mathrm{~m} / \mathrm{s}$, omission of gas phase resistance is 
acceptable. This means EBRT values below 0.3 seconds for the experimental system used in this work. This condition is not fulfilled in the vast majority of biofilters or biotrickling filters [19]. For this reason, experimental values were compared to existing correlations for individual mass transfer coefficients calculation.

Commonly used correlations found in literature for individual mass transfer coefficients calculation were applied. Onda's [8] correlation has been widely used in biofilters and biotrickling filters modeling. It assumes that the wetted surface of each packing element is equal to the effective gas-liquid interface. Van Krevelen and Hoftijzer [20] derived their mass transfer parameters (interfacial areas and coefficients) by means of coupling gas-liquid chemical reactions and absorption for different classical packing materials. Shulman et al. [21] correlation has not been extensively used as the formers. However, it was selected since the authors performed one of the most exhaustive research works in packed columns characterization. In addition Kim and Deshusses [10,13] work was also included since this is (to our knowledge) the only existing paper related to mass transfer coefficients determination for biofilters and biotrickling filters.

Table 3 shows the numerical formulation of the correlations tested. Despite Kim and Deshusses equations seem to be quite different from the rest, their constants are in fact physical parameter groupings. It is noteworthy that the relationship between mass transfer coefficient and gas flowrate is performed by potential equations with exponents quite different from unity. This is not the case of the relationships found for the same variables in the present study (Table 2). On the other hand, effective specific interfacial area and wetted specific surface area were considered the same as values listed in table 1.

Mass transfer coefficients obtained from empirical correlations listed in table 3 are individual ones. Therefore, in order to compare to the experimental values obtained in this work, the following well-known expression was used:

$\frac{1}{\mathrm{HK}_{\mathrm{G}} \mathrm{a}}=\frac{1}{\mathrm{~K}_{\mathrm{L}} \mathrm{a}}=\frac{1}{\mathrm{k}_{\mathrm{L}} \mathrm{a}}+\frac{1}{\mathrm{Hk}_{\mathrm{G}} \mathrm{a}}$ 
Operating conditions for the empirical equations calculations were the same as for the experiments described previously. Figure 6 shows a comparison of the experimental data obtained versus the results provided by the correlations values (theoretical individual mass transfer coefficient values are showed in table 4). For clay pellets (figure 6a) there is clearly an overestimation of the global mass transfer coefficient $\left(\mathrm{K}_{\mathrm{G}} \mathrm{a}\right)$ by the Shulman et al. correlation. In addition, this correlation seems to be hardly sensitive to the EBRT changes. The explanation of this lies on the fact that the individual mass transfer coefficient for the liquid phase is quite low (table 4). This means that the resistance is located mainly in the liquid film. Therefore, the second term of the right-hand side of equation (17) is almost negligible. Since a fixed liquid flowrate was used for all the experimental tests, a quasi-constant value is obtained for the global mass transfer coefficient. The same explanation could be derived for Onda's and Kim \& Deshusses correlation. Apparently, only Van Krevelen \& Hoftijzer correlation seemed to describe moderately well the experimental behavior, despite the predictions failed at low EBRTs.

Figure $6 \mathrm{~b}$ shows the comparison for polyurethane foam. In this case, some trends are also repeated regarding to Onda's and Shulman et al, correlations. In addition, Kim \& Deshusses correlation seems to follow similar behavior as Onda's. Their predictions are strongly affected by the liquid phase resistance and, thus, almost no variation is observed as gas flowrate is increased. Again, the Van Krevelen \& Hoftijzer correlation seems the most suitable despite deviations are important at low EBRT. In any case, predicted values are clearly far from the experimental values even at high EBRT values.

Lava rock comparison (figure $6 \mathrm{c}$ ) shows clearly that no empirical correlation is able to acceptably predict the experimental behavior of the trickling filter. In this case, almost all correlations are insensitive to gas-phase velocity changes, excepting Van Krevelen \& Hoftijzer correlation that shows a slight increase at low EBRT values. However, the deviations are very important. Only at high EBRTs the predicted values are considerably close to the experimental data.

Correlations predictions for Dixon rings (figure 6d) are in concordance with the other materials. While Shulman et al. correlation overpredicts most of the global mass transfer coefficient values, the rest of correlations are only suitable for EBRT above 40 
seconds. Again Shulman et al. and Onda's correlations seem to be insensitive to system conditions changes due to the effect of the liquid phase resistance predicted by their respective equations.

These results show that most of the correlations for mass transfer resistance for the liquid phase tend to overpredict its effect. However, there is no clear frontier or range of values to establish a general rule for neglecting the liquid side mass transfer resistance. Neglecting the liquid phase mass transfer resistance for the theoretical calculations did not give much improvement on the predictions. This confirms that using experimental global mass transfer coefficients appears as the most suitable way to represent mass transfer in biotrickling filter systems.

In order to confirm the correlations limitations stated above, figure 7 shows a comparison of the experimental and calculated values for the gas phase mass transfer coefficient for the four packing materials. For a better comparison, a $\pm 25 \%$ deviation of the diagonal (perfect fitting) is shown with dotted lines. Clearly, the Shulman et al. correlation overpredicts the values, being also hardly sensitive to the experimental values changes. Regarding to Onda's and Kim \& Deshusses correlations, it is also noticeable that they underestimate the experimental values for the coefficient. Only the lowest values are partially well-predicted by either Van Krevelen \& Hoftijzer or Kim \& Deshusses correlations.

\section{CONCLUSIONS}

In this paper, a simple methodology for experimental determination of overall mass transfer coefficients in biotrickling filters has been presented. Results obtained showed only slight differences between packing materials, even if bed porosities were quite different. Existing mathematical correlations for local mass transfer coefficients failed to predict the experimental data obtained. This confirms that when dealing with mass transfer resistance studies (e.g. modeling), the use of empirical correlations is under suspect. Instead, experimental data straightforwardly fitted to simple mathematical expressions are recommended.

\section{ACKNOWLEDGEMENTS}


Antonio David Dorado has received a pre-doctoral scholarship of the MEC (Ministerio de Educación y Ciencia, Spain). Authors acknowledge the financial support provided by the "Comisión Interministerial de Ciencia y Tecnología”' (CICYT), project CTQ 200614997-C02-02. The Department of Chemical Engineering at UAB is a unit of Biochemical Engineering of the Xarxa de Referència en Biotecnologia de Catalunya (XRB), Generalitat de Catalunya.

\section{SYMBOLS USED}

\begin{tabular}{|c|c|c|}
\hline $\mathrm{a}$ & {$\left[\mathrm{m}^{2} / \mathrm{m}^{3}\right.$ reactor $]$} & specific interfacial area \\
\hline$a_{e}$ & {$\left[\mathrm{~m}^{2} / \mathrm{m}^{3}\right.$ reactor $]$} & effective specific interfacial area \\
\hline$a_{p}$ & {$\left[\mathrm{~m}^{2} / \mathrm{m}^{3}\right.$ reactor $]$} & packing specific surface area \\
\hline $\mathrm{a}_{\mathrm{w}}$ & {$\left[\mathrm{m}^{2} / \mathrm{m}^{3}\right.$ reactor $]$} & wetted specific surface area \\
\hline $\mathrm{b}_{1}$ & {$\left[1 / \mathrm{s}^{2}\right]$} & empirical coefficient in eq. (8) \\
\hline $\mathrm{b}_{2}$ & {$[-]$} & empirical coefficient in eq. (8) \\
\hline $\mathrm{C}_{2}, \mathrm{C}_{3}$ & {$[-]$} & empirical coefficients in eq. (15) and (16) \\
\hline $\mathrm{C}$ & {$\left[\mathrm{mol} / \mathrm{m}^{3}\right]$} & toluene concentration \\
\hline $\mathrm{C}^{*}$ & {$\left[\mathrm{~mol} / \mathrm{m}^{3}\right]$} & interfacial toluene concentration \\
\hline$d_{c}$ & {$[\mathrm{~m}]$} & column inner diameter \\
\hline$d_{p}$ & {$[\mathrm{~m}]$} & particle diameter \\
\hline $\mathrm{D}$ & {$\left[\mathrm{m}^{2} / \mathrm{s}\right]$} & diffusion coefficient \\
\hline EBRT & [s] & empty bed residence time \\
\hline g & {$\left[\mathrm{m} / \mathrm{s}^{2}\right]$} & gravitational constant \\
\hline $\mathrm{H}$ & {$[-]$} & dimensionless Henry constant \\
\hline $\mathrm{i}_{2}, \mathrm{i}_{3}$ & {$[-]$} & empirical coefficients in eq. (15) and (16) \\
\hline $\mathrm{k}$ & {$[\mathrm{m} / \mathrm{s}]$} & local or individual mass transfer coefficient \\
\hline K & {$[\mathrm{m} / \mathrm{s}]$} & overall mass transfer coefficient \\
\hline Q & {$\left[\mathrm{m}^{3} / \mathrm{s}\right]$} & flow rate \\
\hline $\mathrm{Sc}$ & {$[-]$} & Schmidt number defined by $\mu / \rho$ D \\
\hline $\mathrm{t}$ & {$[\mathrm{s}]$} & time \\
\hline $\mathrm{u}$ & {$[\mathrm{m} / \mathrm{s}]$} & superficial velocity \\
\hline $\mathrm{V}_{\mathrm{c}}$ & {$\left[\mathrm{m}^{3}\right]$} & packing volume \\
\hline
\end{tabular}

Greek letters

$\varepsilon$

fractional void space of packing 
sphericity factor

$\rho$

$\left[\mathrm{kg} / \mathrm{m}^{3}\right]$

density

$\mu$

$[\mathrm{kg} /(\mathrm{m} \mathrm{s})]$

viscosity

\section{Subscripts}

G

in

L

out

gas

inlet

liquid

outlet 


\section{REFERENCES}

[1] J.S. Devinny, M.A. Deshusses, T.S. Webster, Biofiltration for air pollution control. CRC Press. Boca Raton, Florida 1999.

[2] H. Li, J.C. Crittenden, J.R. Mihelcic, H. Hautakangas, Water Environ. Res. 2002, 74 (1), 5 .

[3] G. Baquerizo, J.P. Maestre, T. Sakuma, M.A. Deshusses, X. Gamisans, D. Gabriel, J. Lafuente, Chem. Eng. J. 2005, 113 (2-3), 205.

[4] A.D. Dorado, G. Baquerizo, J.P. Maestre, X. Gamisans, D. Gabriel, J. Lafuente, Chem. Eng. J. 2008, 140 (1-3), 52.

[5] Md. Amanullah, S. Farooq, S. Viswanathan, Ind. Eng. Chem. Res. 1999, 38 (7), 2765 .

[6] D.S. Hodge, J.S. Devinny, J. Environ. Eng. 1995, 121 (1), 21.

[7] S.J. Hwang, H.M. Tang, W.C. Wang, Environ. Prog. 1997, 16 (3), 187.

[8] K. Onda, H. Takeuchi, Y. Okumoto, J. Chem. Eng. Jpn. 1968, 1, 56.

[9] C.J. Mpanias, B.C. Baltzis, Biotechnol. Bioeng. 1998, 59 (3), 328.

[10] S. Kim, M.A. Deshusses, Chem. Eng. Sci. 2008, 63 (4), 856.

[11] A. Hoffmann, J.F. Mackowiak, A. Górak, M. Haas, J.-M. Löning, T. Runowski, K. Hallenberger, Chem. Eng. Res. Des. 2007, 85 (A1), 40.

[12] G.Q. Wang, X.G. Yuan, K.T. Yu, Ind. Eng. Chem. Res. 2009, 44 (23), 8715.

[13] S. Kim, M.A. Deshusses, Chem. Eng. Sci. 2008, 63 (4), 841. 
[14] F. Heymes, P.M. Demoustier, F. Charbit, J.L. Fanlo, P. Moulin, Chem. Eng. Sci. 2006, 61 (15), 5094.

[15] D. Gabriel, M.A. Deshusses, P. Natl. Acad. Sci. USA. 2003, 100 (11), 6308.

[16] TMECC, Test Methods for the Examination of Composting and Compost. The US composting council research an education foundation, and the US department of Agriculture 2002.

[17] O.J. Prado, R.M. Redondo, J. Lafuente, D. Gabriel, J. Environ. Eng. 2009, 135 (5), 359.

[18] J.L. Rejl, V. Linek, T. Moucha, L. Valenz, Chem. Eng. Res. Des. 2009, 87 (5), 695.

[19] R. Iranpour, H.H.J. Cox, M.A. Deshusses, E.D. Schroeder, Env. Prog. 2005, 24 (3), 254.

[20] D.W. Van Krevelen, P.J. Hoftijzer, Chem. Eng. Prog. 1948, 44 (7), 529.

[21] H.L. Shulman, C.F. Ullrich, A.Z. Proulx, J.O. Zimmerman, AICHE J. 1955, 1, 253. 


\section{FIGURES LEGEND}

Figure 1: Experimental setup of the lab-scale plant; 1: mass flow controller; 2: humidification column; 3: mix chamber; 4: toluene injection; 5: VOC's detector; 6: fixed bed; 7: peristaltic pump; 8:storage tank; 9:control computer A: sample port for liquid inlet; B: sample port for liquid outlet; C: sample port for gas outlet; D: sample port for gas inlet.

Figure 2: Conceptual diagram of the pollutant interfacial mass transfer along the biofilter height.

Figure 3: Experimental raw data example for polyurethane foam with initial toluene concentration $0.0658 \mathrm{~mol} / \mathrm{m}^{3}$ and EBRT $=35 \mathrm{~s}$.

Figure 4: Numerical data handling for global mass transfer coefficients determination. Raw data corresponding to figure 3 .

Figure 5: Experimental values for the global mass transfer coefficients at different empty bed residence times.

Figure 6: Comparison between experimental data and theoretical (empirical correlations) values. a) Clay pellets, b) Polyurethane foam, c) Lava rock, d) Dixon rings.

Figure 7: Comparison of the experimental and calculated values for the gas phase mass transfer coefficient for the four packing materials. 


\section{TABLES LEGEND}

Table 1. Packing materials main characteristics.

Table 2. Empirical coefficients and determination coefficient for equation 8.

Table 3. Empirical correlations for the theoretical individual mass transfer coefficients calculation.

Table 4: Individual mass transfer coefficients for packing materials based on empirical equations calculations. 
Table 1

\begin{tabular}{lccccc}
\hline Material & Shape & $\begin{array}{c}\text { Bed } \\
\text { Porosity } \\
(-)\end{array}$ & $\begin{array}{c}\text { Specific } \\
\text { surface } \\
\mathbf{a r e a} \\
\left(\mathbf{m}^{\mathbf{2}} / \mathbf{m}^{\mathbf{3}}\right)\end{array}$ & $\begin{array}{c}\text { Density } \\
\left(\mathbf{k g} / \mathbf{m}^{\mathbf{3}}\right)\end{array}$ & $\begin{array}{c}\text { Water holding } \\
\text { capacity } \\
(\mathbf{g} / \mathbf{g})\end{array}$ \\
\hline PUF & Cube & 0.92 & 6000.0 & 30.0 & 1.56 \\
Clay Pellets & Sphere & 0.38 & 1375.7 & 388.6 & 2.18 \\
Lava rock & Irregular & 0.50 & 376.9 & 780.3 & 0.18 \\
Dixon rings & Cylinder & 0.74 & 1524.5 & 390.9 & 0.26 \\
\hline
\end{tabular}


Table 2

\begin{tabular}{cccc}
\hline Packing & $\mathbf{b}_{\mathbf{1}}$ & $\mathbf{b}_{\mathbf{2}}$ & $\mathbf{r}^{\mathbf{2}}$ \\
\hline PUF & 6370 & $-1,08$ & 0,986 \\
Clay Pellets & 2941 & $-0,95$ & 0,994 \\
Lava rock & 4456 & $-1,04$ & 0,991 \\
Dixon rings & 14949 & $-1,29$ & 0,986 \\
\hline
\end{tabular}


Table 3

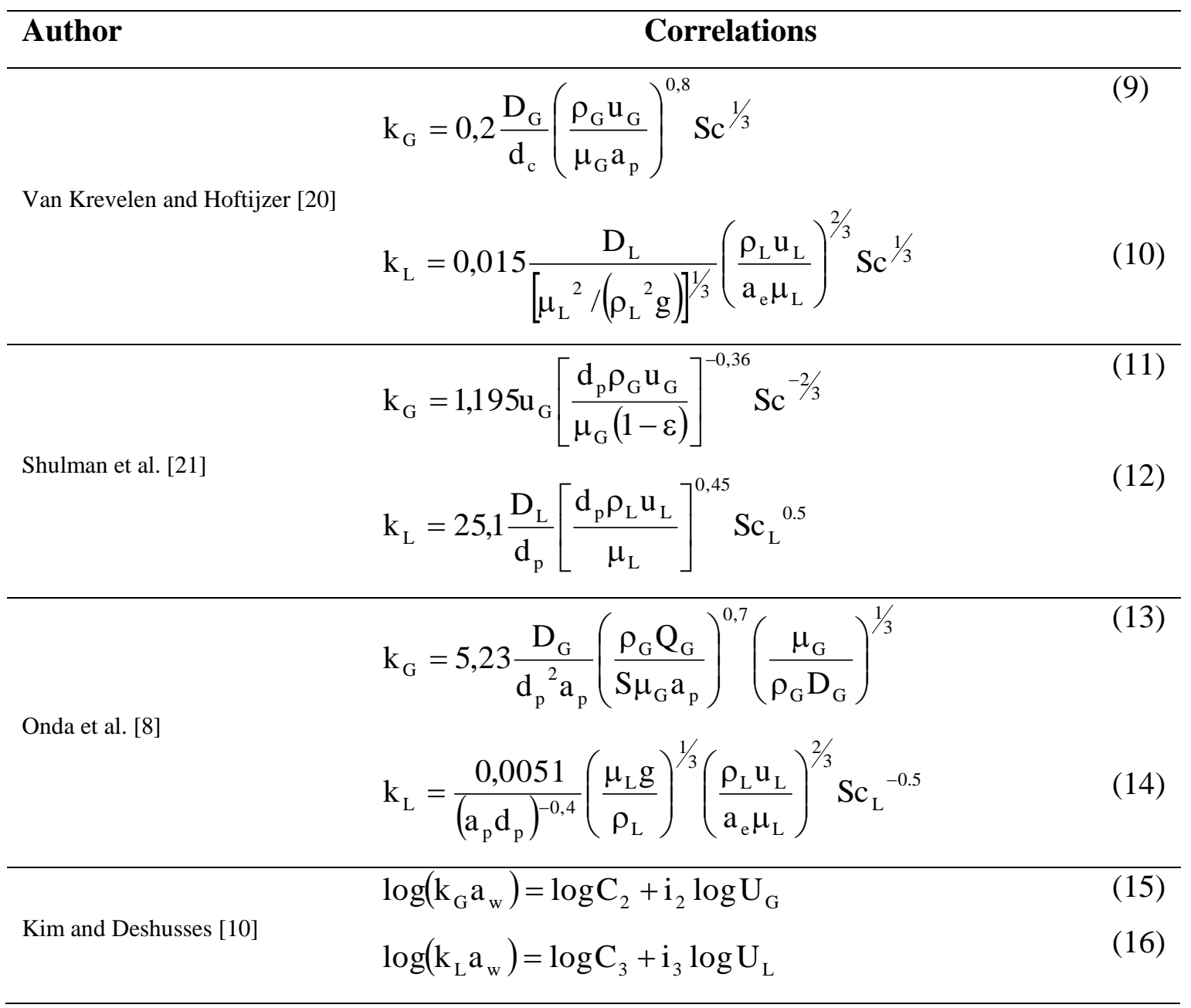




\begin{tabular}{|c|c|c|c|c|c|c|c|c|c|}
\hline & \multirow{2}{*}{ EBRT (s) } & \multicolumn{2}{|c|}{ Onda et al. } & \multicolumn{2}{|c|}{ Van Krevelen } & \multicolumn{2}{|c|}{ Shulman et al. } & \multicolumn{2}{|c|}{ Kim \& Deshusses } \\
\hline & & $k_{G}(m / s)$ & $k_{L}(m / s)$ & $k_{G}(m / s)$ & $k_{L}(m / s)$ & $k_{G}(m / s)$ & $k_{L}(m / s)$ & $k_{G}(m / s)$ & $k_{L}(m / s)$ \\
\hline \multirow{6}{*}{ 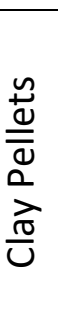 } & 6.48 & $4.68 x^{-7}$ & \multirow{6}{*}{$7.08 x^{-7}$} & $5.37 x^{-7}$ & & $7.93 x^{-3}$ & \multirow{6}{*}{$3.99 x^{-5}$} & - & - \\
\hline & 12.96 & $2.88 x-{ }^{7}$ & & $3.09 x^{-7}$ & & $5.09 x^{-3}$ & & - & - \\
\hline & 38.12 & $1.35 x^{-7}$ & & $1.30 x^{-7}$ & & $2.55 x^{-3}$ & & - & - \\
\hline & 61.71 & $9.66 x^{-8}$ & & $8.86 x^{-8}$ & $4.33 x^{4}$ & $1.87 x^{-3}$ & & - & - \\
\hline & 81.00 & $7.98 x^{-8}$ & & $7.13 x^{-8}$ & & $1.58 x^{-3}$ & & - & - \\
\hline & 99.69 & $6.90 x^{-8}$ & & $6.04 x^{-8}$ & & $1.38 x^{-3}$ & & - & - \\
\hline \multirow{6}{*}{ 岁 } & 8.79 & $2.27 x^{-1}$ & \multirow{6}{*}{$2.22 x^{-6}$} & $7.65 x^{-5}$ & \multirow{6}{*}{$2.70 x^{-2}$} & $6.00 x^{-3}$ & & $1.65 x^{-4}$ & \multirow{6}{*}{$2.83 x^{-6}$} \\
\hline & 17.58 & $1.40 x^{-1}$ & & $4.39 x^{-5}$ & & $3.85 x^{-3}$ & & $1.46 x^{-4}$ & \\
\hline & 35.16 & $8.61 x^{-2}$ & & $2.52 x^{-5}$ & & $2.47 x^{-3}$ & $807 y^{-5}$ & $1.29 x^{-4}$ & \\
\hline & 58.60 & $6.02 x^{-2}$ & & $1.68 x^{-5}$ & & $1.78 x^{-3}$ & $8.07 x$ & $1.17 x^{-4}$ & \\
\hline & 76.43 & $5.00 x^{-2}$ & & $1.36 x^{-5}$ & & $1.50 x^{-3}$ & & $1.12 x^{-4}$ & \\
\hline & 92.53 & $4.37 x^{-2}$ & & $1.16 x^{-5}$ & & $1.33 x^{-3}$ & & $1.08 x^{-4}$ & \\
\hline \multirow{6}{*}{ 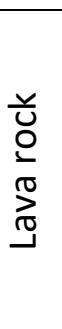 } & 5.22 & $1.40 x^{-8}$ & \multirow{6}{*}{$1.06 x^{-6}$} & $3.62 x^{-7}$ & \multirow{6}{*}{$3.12 x^{-4}$} & $4.53 x^{-3}$ & & $8.39 x^{-7}$ & \multirow{6}{*}{$1.97 x^{-8}$} \\
\hline & 10.44 & $8.63 x^{-9}$ & & $2.08 x^{-7}$ & & $2.91 x^{-3}$ & & $7.35 x^{-7}$ & \\
\hline & 20.88 & $5.31 x^{-9}$ & & $1.19 x^{-7}$ & & $1.86 x^{-3}$ & & $6.44 x^{-7}$ & \\
\hline & 34.80 & $3.71 x^{-9}$ & & $7.94 x^{-8}$ & & $1.34 x^{-3}$ & $1.92 x^{-5}$ & $5.85 x^{-7}$ & \\
\hline & 65.25 & $2.39 \mathrm{E}^{-9}$ & & $4.80 x^{-8}$ & & $8.99 x^{-4}$ & & $5.19 x^{-7}$ & \\
\hline & 80.31 & $2.07 x^{-9}$ & & $4.07 x^{-8}$ & & $7.87 x^{-4}$ & & $4.99 x^{-7}$ & \\
\hline \multirow{6}{*}{ 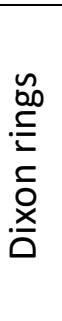 } & 5.61 & $4.00 x^{-3}$ & \multirow{6}{*}{$2.83 x^{-6}$} & $3.63 x^{-5}$ & & $5.92 x^{-3}$ & \multirow{6}{*}{$1.45 x^{-2}$} & - & - \\
\hline & 11.22 & $2.46 x^{-3}$ & & $2.08 x^{-5}$ & & $3.80 x^{-3}$ & & - & - \\
\hline & 22.44 & $1.52 x^{-3}$ & & $1.20 x^{-5}$ & & $2.44 x^{-3}$ & & - & - \\
\hline & 59.05 & $1.06 x^{-3}$ & & $7.95 x^{-6}$ & $2.83 x^{-6}$ & $1.76 x^{-3}$ & & - & - \\
\hline & 78.00 & $7.70 x^{-4}$ & & $5.52 x^{-6}$ & & $1.31 x^{-3}$ & & - & - \\
\hline & 93.60 & $5.58 x^{-4}$ & & $3.82 x^{-6}$ & & $9.79 x^{-4}$ & & - & - \\
\hline
\end{tabular}




\title{
SHORT TEXT FOR THE TABLE OF CONTENTS
}

\author{
ADD FIGURE 5
}

A simple methodology for overall mass transfer coefficients determination was developed for gas-phase biofilters. Important discrepancies between experimental data and empirical correlations commonly used in studies related to modeling of gas-phase biotrickling filters have arisen. The study has shown that operating conditions influence dramatically the suitability of such correlations. 